\section{A Comparative Study on Stabilization of Chromite Ore Processing Residue with Prevalent Reductants}

TATHAGATA BANDYOPADHYAY, SUMIT KUMAR, MAINAK BHATTACHARYA AND ABHAS SINGH

Indian Institute of Technology Kanpur

Presenting Author: tathabd@iitk.ac.in

Chromite ore processing residue (COPR) is generated during production of basic chromium sulphate by high temperature lime roasting process. COPR contains significant amounts of hexavalent chromium, $\mathrm{Cr}(\mathrm{VI})$, which is a confirmed carcinogen. Unregulated disposal of this hazardous solid waste is a serious environmental concern, as it leaches $\mathrm{Cr}(\mathrm{VI})$ in groundwater upon weathering. The goal of this study was to engineer an efficient stabilization method of a large COPR heap accumulated over more than 30 years of dumping near Kanpur, India. This was achieved through: (a) extensive sampling to characterize the waste pile; and (b) by bench- and pilot- scale remediation experiments. Solid samples were collected depth-wise contained particles of varied sizes, ranging from $\sim 2-800 \mathrm{~mm}$. The solids were characterized by X-ray diffraction (XRD) to identify the minerals present in them (Fig. 1(a)). COPR samples were also digested to quantify total $\mathrm{Cr}$ and $\mathrm{Cr}(\mathrm{VI})$ using established protocols. Results showed remarkably high contamination levels compared to that reported in literature $(>7 \%$ total $\mathrm{Cr}$ and $>40 \%$ $\mathrm{Cr}(\mathrm{VI})$ of total $\mathrm{Cr}$ for some samples). Leaching experiments were performed in ultrapure water on a $\mathrm{pH}$ range of 4-11 to understand the processes controlling $\mathrm{Cr}(\mathrm{VI})$ mobilization (Fig.1(b)). As the efficiency of most reductants was known to depend on $\mathrm{pH}$, proton requirements (eq $\mathrm{kg}^{-1}$ ) were identified from alkalinity tests. A series of bench-scale studies was performed to identify the best reductant among five reductants - iron (II) sulphate, sodium metabisulphite, sodium hydrosulphite, calcium polysulfide, and a compost leachate. The experiments were performed with an L:S of 1 and at a $\mathrm{pH}$ of 8.5-9.5. Variable dosages of the reductants were attempted to identify the most optimal dosage. These solids were then cured for a period of 30 $\mathrm{d}$, during which TCLP extraction was performed at predetermined intervals to identify the best treatment time. The results from these experiments will be used to identify the best reductant in terms of $\mathrm{Cr}$ leaching potential, economy, time and ease of handling in pilot-scale studies. Besides stabilization of the mentioned dumpsite, the results of this study could have implications for the stabilization and remediation of other COPR heaps worldwide.
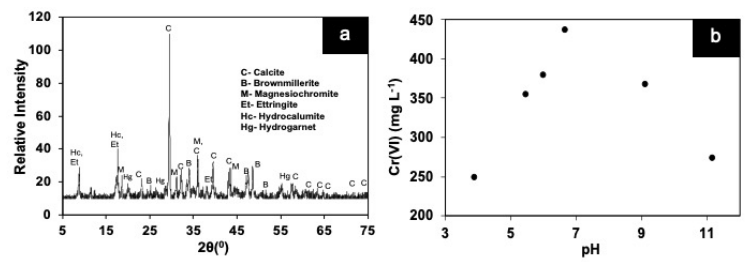

Figure 1. (a) X-ray diffractogram of samples showed peaks of magnesiochromite (raw $\mathrm{Cr}$ ore) and $\mathrm{Cr}(\mathrm{VI})$-bearing hydrogarnet and hydrocalumite. (b) Results from COPR leaching in ultrapure water (L:S = 20) for varying amounts of acid. 